\title{
Dynamic SIMS Applications for Photovoltaic Technology Development
}

\author{
A.N. Davis*, P. Peres**, A. Merkulov**, F. Desse**, S-Y. Choi**, M. Schuhmacher** \\ * Cameca Instruments Inc., 91 McKee Drive, Mahwah, NJ 07430, USA \\ ** Cameca, 29 quai des Grésillons, 92622 Gennevilliers Cedex, France
}

SIMS (Secondary Ion Mass Spectrometry) technique is widely applied to IC development and process control. The solar photovoltaic (PV) cell technology shares many of the processes and materials used for semiconductor device manufacturing.

The advantages of SIMS analytical technique for semiconductor applications are well established: extreme sensitivity, high mass resolution, high dynamic range and low detection limits while keeping high analysis throughput. Thus, over the years, interest in the SIMS technique for solar PV cell development has been growing significantly.

Research for manufacturing low-cost PV cells brings a renewed interest for SIMS in the quality control of materials. For example, in UMG-Si ingots, depth profiles can be recorded up to several microns within minutes, while offering detection limits from the ppm down to the ppb range (depending on the species to be analyzed). Nearly all species in the periodic table can be analyzed: light elements H, C, F, O; main Si dopants B, P, As; metals such as Al, Cr, Fe, Ni, Cu, etc.).

SIMS imaging capability can also be used to investigate local non-uniformity of trace elements at the micron scale. Application examples of bulk analysis of Si will be presented to highlight the recent developments of dynamic SIMS tools, targeted to improve analytical capability for trace element analysis in bulk samples.

Research for increasing the yield of PV cells is based on multi-layer structure development. For instance, the development of CIGS thin films requires investigations of the interface quality and diffusion of contaminants between the different, thin layers. We will present results for such device characterization, therefore addressing the data quantification issue in these multi-matrix structures, especially using the "MCs+ "analytical technique. 